\title{
Noise in MgO barrier magnetic tunnel junctions with CoFeB electrodes: Influence of annealing temperature
}

\author{
J. Scola, ${ }^{\text {a) }}$ H. Polovy, C. Fermon, and M. Pannetier-Lecœur \\ Service de Physique de L'État Condensé, DSM/DRECAM/SPEC, CEA-Saclay, 91191 Gif-sur-Yvette, France \\ G. Feng, K. Fahy, and J. M. D. Coey \\ CRANN, Trinity College, Dublin 2, Ireland and School of Physics, Trinity College, Dublin 2, Ireland
}

(Received 16 April 2007; accepted 24 May 2007; published online 18 June 2007)

\begin{abstract}
Low frequency noise has been measured in magnetic tunnel junctions with $\mathrm{MgO}$ barriers and magnetoresistance values up to $235 \%$. The authors investigated the noise for different degrees of crystallization and $\mathrm{CoFeB} / \mathrm{MgO}$ interface quality depending on the annealing temperature. The authors report an extremely low $1 / f$ noise, compared to magnetic junctions with $\mathrm{Al}_{2} \mathrm{O}_{3}$ barriers. The origin of the low frequency noise is discussed and it is attributed to localized charge traps with the MgO barriers. () 2007 American Institute of Physics. [DOI: 10.1063/1.2749433]
\end{abstract}

The resistance of a magnetic tunnel junction (MTJ) depends on the relative magnetization of the ferromagnetic layers: it is usually low in the parallel configuration (PC) and high in the antiparallel configuration (APC). This effect is used for storage in magnetic random access memory and is now being adopted for read heads in place of giant magnetoresistance (GMR) sensors. The prediction ${ }^{1,2}$ and recent observation $^{3}$ of tunnel magnetoresistance (TMR) ratios $\left[\left(R_{\mathrm{AP}}-R_{\mathrm{P}}\right) / R_{\mathrm{AP}}\right]$ as high as $1000 \%$ in MTJs with $\mathrm{MgO}$ barrier make those devices very attractive candidates to outclass GMR in high-resolution sensors. However, a high TMR ratio is not enough to make a good magnetoresistive sensor, since its performance is determined by the sensitivity which is the output voltage per unit field divided by the voltage noise.

The noise level is as important as the magnitude of the signal itself. From earlier studies on MTJs with $\mathrm{Al}_{2} \mathrm{O}_{3}$ barriers, the noise can be traced back to three sources. The first is the frequency-independent thermal noise, which is an intrinsic property of a tunnel junction that defines the ultimate noise limit. Here, we will focus on the two other contributions to the noise, which may be reduced or even eliminated provided the appropriate parameters are known. At low frequencies, noise is dominated by a $1 / f$ component, which is analogous to $1 / f$ noise in conductors. It is quantified by the Hooge-like parameter $\alpha=A f S_{V}(f) / V^{2}$, expressed in units of area; $A$ is the surface area of the junction, $S_{V}(f)$ the noise power spectrum, and $V$ the voltage drop across the tunnel barrier. Its origin is not fully understood, and, in particular, the influence of magnetization is still unclear although it is commonly observed that $\alpha$ is higher in the APC than in the PC. ${ }^{4,5}$ The quality of the metal-barrier interface may also play a role in the $1 / f$ noise. ${ }^{4}$ The third component in MTJs is random telegraphic noise (RTN), namely, discrete fluctuators in the voltage-time traces associated with a Lorentzian-like spectrum. It is characterized by a strong sample-to-sample random of its amplitude and switching rate and it is interpreted either in terms of charge trapping or magnetic domain fluctuations. ${ }^{6}$

In this letter, we discuss low frequency noise properties of MTJs with a $\mathrm{MgO}$ barrier which exhibit TMR ratios up to

\footnotetext{
${ }^{a)}$ Electronic mail: joseph.scola@cea.fr
}

$235 \%$. Our focus is on the annealing temperature, which is the crucial parameter for a high TMR ratio. We discuss the origins of the noise, based on calculated transport properties; ${ }^{1,2}$ those calculations have been rendered possible by the crystalline structure of $\mathrm{MgO}$ and could not have been performed for amorphous $\mathrm{Al}_{2} \mathrm{O}_{3}$ barriers.

Our MTJs consist of Ta $5 / \mathrm{Ru} 50 / \mathrm{Ta} 5 / \mathrm{NiFe} 5 / \mathrm{IrMn}$ $10 / \mathrm{CoFe} \quad 2 / \mathrm{Ru} \quad 0.7 / \mathrm{CoFeB} \quad 3 / \mathrm{MgO} \quad 2.5 / \mathrm{CoFeB} \quad 5 / \mathrm{NiFe}$ $5 /$ Ta 5 multilayer stacks grown by magnetron sputtering (the order from the bottom electrode to the top with thickness being given in nanometers). Further details on the growth process can be found in Ref. 7. The multilayer stacks are patterned into $16 \times 16$ and $24 \times 24 \mu \mathrm{m}^{2}$ junctions by UV lithography (series A and B, respectively), then annealed in $0.8 \mathrm{~T}$ for 90 min at $250{ }^{\circ} \mathrm{C}(\mathrm{B} 2), 300{ }^{\circ} \mathrm{C}(\mathrm{B} 3)$, and $375^{\circ} \mathrm{C}$ (A and B4). MTJ B1 is not annealed. Annealing aims at improving the degree of crystallization of the ferromagnetic layer that is amorphous as deposited: the $\Delta_{1}$ band responsible for a good band matching is created by annealing, thus yielding high TMR ratios. ${ }^{2}$ If the temperature is further increased, the MR drops because of diffusion of $\mathrm{Ru}$ and $\mathrm{Mn}$ atoms from the artificial antiferromagnetic layer ruining the exchange bias. Furthermore, annealing also improves the quality of the $\mathrm{CoFeB} / \mathrm{MgO}$ interface as it has been suggested experimentally in Ref. 7. Noise measurements were performed in a cryostat at 300, 77, and $4 \mathrm{~K}$. We used a INA103 preamplifier in an electronic circuit optimally designed for our MTJs resistances (ranging from 1 to $20 \mathrm{k} \Omega$ ), yielding a background noise of $3 \mathrm{nV} / \sqrt{\mathrm{Hz}}$. The amplified signals are digitalized at $50 \mathrm{kHz}$ by a 16 data bit acquisition card and numerically processed. All noise data are measured at zero magnetic field, the magnetic configuration having been set by applying a saturating field and switching it off.

Let us consider first the noise signature of the junction A, which was annealed at $T_{a}=375^{\circ} \mathrm{C}$ and presents a TMR ratio of $235 \%$. Its ferromagnetic layers are well crystallized, and the $\mathrm{CoFeB} / \mathrm{MgO}$ interface is sharp. In Fig. 1, we show the power spectral densities $S_{V}(f)$ for several bias currents at $77 \mathrm{~K}$. High frequency noise is dominated by a frequencyindependent component that corresponds to the thermal noise, indicated by the dashed lines in the graph. At low frequency, $1 / f$ noise is observed. We measured room temperature values of $\alpha$ of the order of $10^{-10} \mu \mathrm{m}^{2}$. As the 


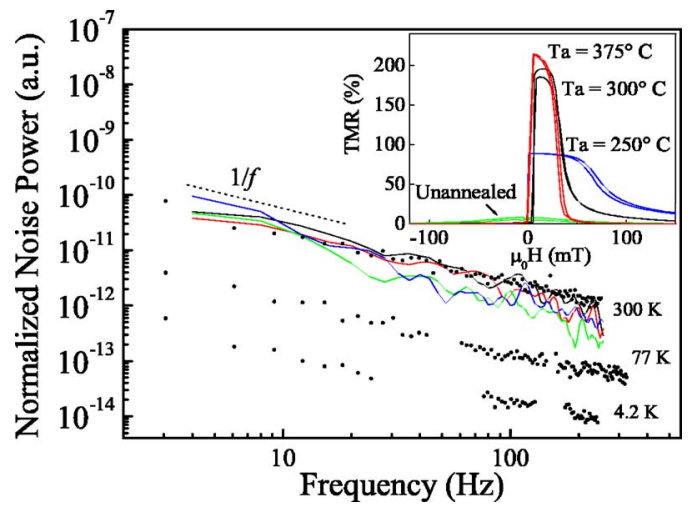

FIG. 1. (Color online) Power spectral densities $\left[S_{V}(f)\right]$ of a $375^{\circ} \mathrm{C}$ annealed MTJ measured at $77 \mathrm{~K}$ in the PC, for $I=0,20$, and $50 \mu \mathrm{A}$, corresponding to $V=0,106$, and $306 \mathrm{mV}$. The dashed lines represent the extrapolation to low frequencies of the white noise that increases with the current. Inset: Bias dependence of the TMR ratio normalized by the zero bias TMR ratio (open circles) and voltage noise at $12 \mathrm{~Hz}$ expressed in $\mathrm{nV} / \mathrm{Hz}^{2}$ in the PC (squares) and APC (triangles) plotted against the voltage. The slope of the solid lines is proportional to $\sqrt{\alpha}$. The dotted line represents the amplifier background noise.

junction is cooled down to $4.2 \mathrm{~K}, \alpha$ decreases by two orders of magnitudes (Fig. 2). It is remarkable that in our MTJs with a $\mathrm{MgO}$ barrier, $\alpha$ is at least two orders of magnitude lower than in $\mathrm{Al}_{2} \mathrm{O}_{3}$ MTJs at a given temperature. ${ }^{4,6,8,9}$ It must also be noted that the $V^{2}$ dependence of the $1 / f$ noise power $S_{V}$ is linear (inset of Fig. 1 displays square-root values). This means that $\alpha$ is practically constant in the explored range of voltages $(V \approx 0-400 \mathrm{mV})$, while the TMR ratio drops by $50 \%$. Also noteworthy is the asymmetry of $\alpha$ depending on the sign of the voltage. The $1 / f$ noise is significantly higher for positive voltages, when the electrons pass from the bottom up to the top electrode, than for negative voltages (Table I). We will discuss these points later.

Now, we focus on the effect of the annealing temperature on the magnetoresistance which is shown in the TMR versus magnetic field curves (inset of Fig. 2). The unannealed junction (B1) exhibits a very low TMR ratio, and in the annealed junctions with $T_{a}$ up to $375^{\circ} \mathrm{C}$ the TMR ratio increases (B2-B4, respectively, MTJ A having similar behavior to B4). We measured the voltage noise at $T=300 \mathrm{~K}$ for different annealing temperatures. The normalized noise power spectra $S_{V}(f) A / V^{2}=\alpha / f$ for MTJs B1-4 are presented

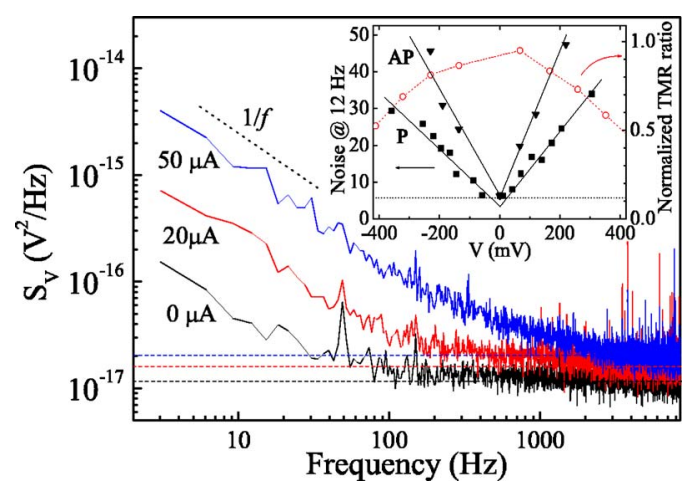

FIG. 2. (Color online) Normalized noise power spectra $S_{V}(f) A / V^{2}=\alpha / f$. MTJ A (dots) at 300, 77, and $4.2 \mathrm{~K}$, for $V=130,306$, and $901 \mathrm{mV}$, respectively. Solid lines are spectra measured at $V=500 \mathrm{mV}$ in three junctions annealed at $T_{a}=250,300,375^{\circ} \mathrm{C}$ (B2-B4) and one unannealed junction (B1). All data are measured in the PC. Inset: TMR ratio vs magnetic field of

junctions B1-B4.
Downloaded 12 Aug 2009 to 134.226.1.229. Redistribution subject to AlP license or copyright; see http://apl.aip.org/apl/copyright.jsp
TABLE I. $1 / f$ noise level represented by $\alpha$ (expressed in $\mu \mathrm{m}^{2}$ ) at different temperatures, for both magnetic configurations and voltage signs. Values are given within $10 \%$ error bars accounting for the statistical dispersion.

\begin{tabular}{cccccc}
\hline \hline & \multicolumn{2}{c}{$\alpha$ parallel } & & \multicolumn{2}{c}{$\alpha$ antiparallel } \\
\cline { 2 - 3 } \cline { 5 - 6 }$T(\mathrm{~K})$ & $V>10$ & $V<0$ & & $V>0$ & $V<0$ \\
\hline 300 & $2.5 \times 10^{-10}$ & & $5.0 \times 10^{-10}$ & \\
77 & $3.2 \times 10^{-11}$ & $2.0 \times 10^{-11}$ & & $1.3 \times 10^{-10}$ & $6.5 \times 10^{-11}$ \\
4.2 & $9.6 \times 10^{-13}$ & $2.7 \times 10^{-13}$ & & $2.8 \times 10^{-12}$ & $3.0 \times 10^{-12}$ \\
\hline \hline
\end{tabular}

in Fig. 2 (solid lines). Interestingly, all spectra lie within the statistical deviation, meaning that the magnitude of the $1 / f$ noise does not vary significantly with $T_{a}$. Hence, it can be directly associated neither with the degree of crystallization of the ferromagnetic electrodes, nor with the quality of the $\mathrm{CoFeB} / \mathrm{MgO}$ interface. Likewise, no correlation can be found between the $1 / f$ noise and the TMR ratio.

We also observe occurrences of RTN in the MTJ annealed at $250{ }^{\circ} \mathrm{C}$ (B2) in addition to the $1 / f$ noise. The switching rate of the associated fluctuator is extremely sensitive to the current and to the applied magnetic field and it is

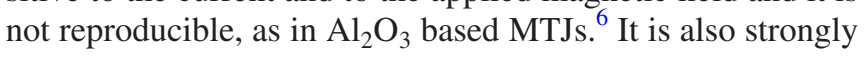
dependent on the temperature: Fig. 3 illustrates the variation of the RTN as the sample is cooled down. Annealing at $250{ }^{\circ} \mathrm{C}$ is not high enough to completely crystallize the ferromagnetic layers as can be seen from the moderate TMR ratio (inset of Fig. 2); some crystallographic defects remain, which is favorable for the creation of magnetic domains. In contrast, we did not measure any RTN in the MTJ annealed at $375{ }^{\circ} \mathrm{C}$, in which the $\mathrm{CoFeB}$ layers are fully, or at least better crystallized. We attribute the RTN to fluctuating magnetic domains in the ferromagnetic electrodes, as was already been proposed in AlO based MTJs. ${ }^{6}$

Although layer magnetization fluctuations seem to be responsible for the RTN, we doubt that they are an ingredient of major importance in the $1 / f$ noise here, despite the dependence of $\alpha$ on the magnetic alignment of the layers. Indeed, resistance fluctuations originating from magnetization fluctuations should strengthen as the TMR ratio increases with $T_{a}$, which is contradicted by our results. Similarly, the dependence of $\alpha$ upon the magnetic alignment (see Table I) should be related to the TMR ratio, whereas $\alpha$ increases in the same proportion (a factor of 2-3) in $\mathrm{Al}_{2} \mathrm{O}_{3}$ MTJs with only $40 \%$ TMR. ${ }^{4,6}$ Instead, we attribute the $1 / f$ noise to charge trap-

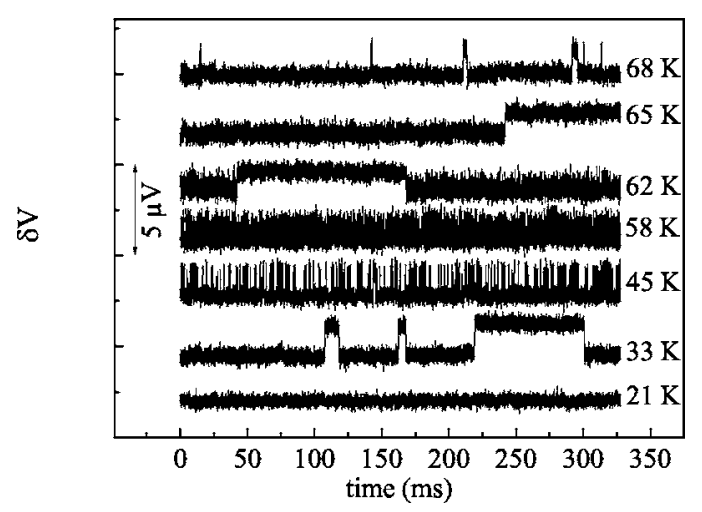

FIG. 3. Voltage fluctuations at different temperatures in the $250{ }^{\circ} \mathrm{C}$ annealed sample. The bias current is kept constant at $I=0.2 \mathrm{~mA}$ which correAlP license or copyright; see http://apl.aip.org/apl/copyright.jsp 
ping in the insulating barrier, as is the case in nonmagnetic tunnel junctions. ${ }^{10}$ The way the electrons tunnel through the barrier depending on the layer magnetization can be considered. The conductivity channels have been detailed in calculations by Butler et $a l^{2}$ It has been shown that conductivity in the PC is dominated by $s$ electrons of the $\Delta_{1}$ band which have small parallel components of their wave vector $\left(k_{\|}\right)$. Minority and antiparallel conductivity are due to localized electrons with a given $k_{\|}$whose density of states decays much faster in the $\mathrm{MgO}$ barrier than the $s$ electrons. The constancy of $\alpha$ regardless of the existence of the $\Delta_{1}$ band (determined by $T_{a}$ ) shows that the contribution of this conductivity channel to the noise is negligible and, consequently, that most of the noise comes from electrons with larger $k_{\|}$ from either majority or minority band. This is coherent with the fluctuations originating from localized charge traps in the insulator (e.g., magnetic impurities) since they are expected to have more effect on localized electrons than on delocalized ones. Since no $s$ electrons can tunnel in the APC, the noise is higher relative to the resistance than in the $\mathrm{PC}$ where a part of the conduction is practically noise-free. Such a noise mechanism is very sensitive to the polarization as can be seen from the asymetry of $\alpha$ with the sign of the voltage (Table I). Depending on whether tunneling electrons come from the thin $(V>0)$ or the thick $\mathrm{CoFeB}$ electrode $(V<0)$, the spin up and spin down populations slightly vary, and so do the conductivity channels, thus leading to different amounts of noise.
In conclusion, the order of magnitude of the Hooge-like parameter $\alpha$, which is as small as $10^{-12} \mu \mathrm{m}^{2}$ at low temperature in our junctions, regardless of the TMR ratio is very promising for low frequency applications. Neither the TMR ratio, nor the $\mathrm{CoFeB} / \mathrm{MgO}$ interface quality nor the $\mathrm{CoFeB}$ crystallization and magnetic stability is a relevant parameter for the $1 / f$ noise in our junctions, which we attribute to charge trapping in the insulating barrier. The random telegraph noise in underannealed junctions is associated with magnetic fluctuations.

This work is supported by the European program ISTNMP-2 Bio-sensors for Diagnosis and Healthcare (Biomagsens NMP4-CT-2005-07210).

${ }^{1}$ J. Mathon and A. Umerski, Phys. Rev. B 63, 220403 (2001).

${ }^{2}$ W. H. Butler, X.-G. Zhang, T. C. Schultless, and J. M. MacLaren, Phys. Rev. B 63, 054416 (2001).

${ }^{3}$ J. Hayakawa, S. Ikeda, Y. M. Lee, F. Matsukara, and H. Ohno Appl. Phys. Lett. 89, 232510 (2006).

${ }^{4}$ E. R. Nowak, M. B. Weissman, and S. S. P. Parkin, Appl. Phys. Lett. 74, 600 (1999).

${ }^{5}$ J. M. Almeida, R. Ferreira, P. P. Freitas, J. Langer, B. Ocker, and W. Maass, J. Appl. Phys. 99, 08B314 (2006).

${ }^{6}$ L. Jiang, E. R. Nowak, P. E. Scott, J. Johnson, J. M. Slaugther, J. J. Sun, and R. W. W. Dave, Phys. Rev. B 69, 054407 (2004).

${ }^{7}$ G. Feng, S. van Dijken, and J. M. D. Coey, Appl. Phys. Lett. 89, 162501 (2006).

${ }^{8}$ W. K. Park, J. S. Moodera, J. Taylor, M. Tondra, J. M. Daughton, A. Thomas, and H. Brückl, J. Appl. Phys. 93, 7020 (2003).

${ }^{9}$ E. R. Nowak, R. D. Merithew, M. B. Weissman, I. Bloom, and S. S. P. Parkin, J. Appl. Phys. 84, 6195 (1998).

${ }^{10}$ C. T. Rogers and R. A. Buhrman, Phys. Rev. Lett. 55, 859 (1985). 